“ C 2019 IEEE. Personal use of this material is permitted. Permission from IEEE must be obtained for all other uses, in any current or future media, including

reprinting/republishing this material for advertising or promotional purposes, creating new collective works, for resale or redistribution to servers or lists, or reuse of any copyrighted component of this work in other works." 


\title{
Angle-of-arrival Acquisition and Tracking via Virtual Subarrays in an Analog Array
}

\author{
Chuan Qin, J. Andrew Zhang, Xiaojing Huang, Y. Jay Guo \\ Global Big Data Technologies Centre, University of Technology Sydney, Australia \\ Email: Chuan.Qin@student.uts.edu.au; \{Andrew.Zhang; Xiaojing.Huang; Jay.Guo\}@uts.edu.au
}

\begin{abstract}
Angle-of-arrival (AoA) estimation is a challenging problem for analog antenna arrays. Typical algorithms use beam scanning and sweeping, which can be time-consuming, and the resolution is limited to the scanning step. In this paper, we propose a virtual-subarray based AoA estimation scheme, which divides an analog array into two virtual subarrays and can obtain a direct AoA estimate from every two temporal measurements. We propose different subarray constructions which lead to different range and accuracy of estimation. We provide detailed beamforming vector designs for these constructions and provide a performance lower bound for the estimator. We also present how to apply the estimator to AoA acquisition and tracking. Simulation results demonstrate that the proposed scheme significantly outperforms existing ones when the signal-to-noise ratio is not very low.
\end{abstract}

\section{INTRODUCTION}

Millimeter wave (mmWave) systems can provide very high data rate and accurate localization for $5 \mathrm{G}$ cellular and vehicular networks. To balance the cost and performance, a basestation will typically use a mmWave Hybrid array, while a mobile user terminal will use a beam-steerable analog array.

For both hybrid and analog arrays, a fundamental and challenging problem in communication and localization is angle-of-arrival (AoA) estimation. For hybrid arrays, the challenge is mainly arisen from the nonlinear nature of the AoA estimation, and for the special localized hybrid array it is also associated with the phase ambiguity problem even in the presence of a single LOS path only [1]. For analog arrays, AoA estimation is typically realized via beam scanning [2], which uses narrow beams to scan the directions of interest and find the AoA via identifying the one with largest received power. Advanced techniques such as multi-resolution sweeping [3] and compressive sensing based random sweeping [4] have also been proposed. However, these methods still cannot directly estimate the AoA and can only get the estimate up to a quantized value, depending on either the scanning interval [3] or the dictionary grid [4].

In this paper, we propose a virtual-subarray based AoA estimation scheme for analog antenna arrays, which can directly estimate the AoA of the dominating LOS path. This method divides the analog array into two virtual subarrays and applies specially designed beamforming (BF) vectors to these subarrays. It then estimates the AoA using a pair of two measurements over the time domain when the channel is assumed to be unchanged. We propose different ways of constructing virtual subarrays, which lead to different ranges and accuracy of AoA estimation. We also provide performance analysis for the estimator by characterizing its Cramer-Rao Lower Bound (CRLB). We also present the ways of applying the estimator to AoA acquisition and tracking. Simulation results are provided and demonstrate that the proposed scheme can achieve much better estimation accuracy than conventional beam scanning and sweeping methods, at very low complexity, when the signal-to-noise ratio (SNR) is not very small.

\section{SySTEM MOdEL}

We consider a uniform linear array (ULA) with $N$ antenna elements, where each element has an adjustable gain and phase shift. The antenna interval is half wavelength.

Typical mmWave channels consists of one dominating lineof-sight (LOS) path and a limited number of non-line-of-sight (NLOS) paths. The received signal at time $t$ is given by

$$
y(t)=\sum_{\ell=1}^{L} d_{\ell} \mathbf{w}^{T}(t) \mathbf{a}\left(\theta_{\ell}\right) s(t)+\eta(t)
$$

where $\eta(t)$ is the additive white Gaussian noise (AWGN) with mean zero and variance $\sigma_{\eta}^{2} ; s(t)$ is the transmitted signal; $L$ denotes the total number of paths, $d_{\ell}$ and $\theta_{\ell}$ are the complex amplitude and the AoA of the $\ell$-th path, respectively; $\mathbf{w}(t)$ is the BF vector and $\mathbf{w}^{H}(t) \mathbf{w}(t)=1$; and $\mathbf{a}\left(\theta_{\ell}\right)$ is the array response vector (steering vector) corresponding to the $\ell$-th multipath

$$
\mathbf{a}\left(\theta_{\ell}\right)=\left[1, e^{j u_{\ell}}, \ldots, e^{j(N-1) u_{\ell}}\right]^{T},
$$

with $u_{\ell}=\pi \sin \left(\theta_{\ell}\right) \in(-\pi, \pi]$ which is called as equivalent AoA here.

Our scheme can be applied to the case when either $s(t)$ is known or unknown but with fixed relationship between any two samples. Here, we simply assume that the two signals are unknown but are repeated over the time of measurements.

\section{Virtual-SubarRay based AoA Estimation}

The basic idea of the proposed scheme is to divide the single analog array into two virtual subarrays and apply carefully designed $\mathrm{BF}$ vectors to them, so that the AoA can be directly estimated using two measurements over the time domain.

Assume that the number of antennas $N$ is an even number. There could be multiple ways of dividing the total $N$ antenna elements into two subarrays. Referring to (2), we want to form two virtual subarrays whose array response vectors differ by a 
TABLE I: Antenna indexes of virtual subarrays for different $Q_{k}$ values.

\begin{tabular}{|c|c|c|}
\hline Value of $Q$ & $\begin{array}{c}\text { Antenna Indexes of } \\
\text { Virtual subarray 1 }\end{array}$ & $\begin{array}{c}\text { Antenna Indexes of } \\
\text { Virtual subarray 2 }\end{array}$ \\
\hline$Q=1$ & $0,2,4,6,8,10,12,14$ & $1,3,5,7,9,11,13,15$ \\
\hline$Q=2$ & $0,1,4,5,8,9,12,13$ & $2,3,6,7,10,11,14,15$ \\
\hline$Q=4$ & $0,1,2,3,8,9,10,11$ & $4,5,6,7,12,13,14,15$ \\
\hline$Q=8$ & $0,1,2,3,4,5,6,7$ & $9,10,11,12,13,14,15$ \\
\hline
\end{tabular}

constant phase shift of $e^{j Q u_{\ell}}$. Using $N=16$ as an example, we can form the first and second virtual subarrays with antenna elements as shown in Table I.

We denote the BF vectors for two subarrays as $\mathbf{w}_{1}(t)$ and $\mathbf{w}_{2}(t)$. Accordingly, we divide the array response vector $\mathbf{a}\left(\theta_{\ell}\right)$ to two parts and denote them as $\mathbf{a}_{1}\left(\theta_{\ell}\right)$ and $\mathbf{a}_{2}\left(\theta_{\ell}\right)$. It is easy to see that $\mathbf{a}_{2}\left(\theta_{\ell}\right)=e^{j Q u \ell} \mathbf{a}_{1}\left(\theta_{\ell}\right)$. This relationship will be exploited for estimating the dominating LOS AoA.

Hereafter, we treat NLOS paths as interference and incorporate them to the noise term. The received signal in (1) can be rewritten as

$$
y(t)=d\left(\mathbf{w}_{1}^{T}(t)+e^{j Q u} \mathbf{w}_{2}^{T}(t)\right) \mathbf{a}_{1}(\theta) s(t)+z(t),
$$

where $z(t)$ denotes the combined noise and NLOS path signals.

Next, we propose a scheme for deriving one estimate for $u$ and then the AoA $\theta$, using two measurements at time $t_{2 k}$ and $t_{2 k+1}$. The channel is assumed to remain unchanged during the period. For simplicity, we use $2 k$ and $2 k+1$ to index the pair of measurements.

\section{A. Estimate $u=\pi \sin (\theta)$}

To get $y(2 k)$ and $y(2 k+1)$, we set the BF vectors

$\mathbf{w}_{2}(2 k)=e^{j \beta_{k}} \mathbf{w}_{1}(2 k)$, and

$\mathbf{w}_{2}(2 k+1)=-e^{j \beta_{k}} \mathbf{w}_{1}(2 k+1)$, and $\mathbf{w}_{1}(2 k+1)=\mathbf{w}_{1}(2 k)$,

where $\beta_{k}$ is a known coefficient. We will discuss how to set the values of $\beta_{k}$ and the BF vectors in Section III-C and III-D.

Let $b_{k}=\mathbf{w}_{1}^{T}(2 k) \mathbf{a}_{1}(\theta)$. The two received signals are given by

$$
\begin{aligned}
& y(2 k)=\left(1+e^{j\left(\beta_{k}+Q_{k} u\right)}\right) b_{k} d s(2 k)+z(2 k), \\
& y(2 k+1)=\left(1-e^{j\left(\beta_{k}+Q_{k} u\right)}\right) b_{k} d s(2 k+1)+z(2 k+1),
\end{aligned}
$$

where we use $Q_{k}$ in place of $Q$ to highlight that the values of $Q$ can be varying with $k$ in our proposed method.

We compute the difference and sum between $y(2 k)$ and $y(2 k+1)$, and obtain

$$
\begin{aligned}
r_{d, k} & =2 e^{j\left(\beta_{k}+Q_{k} u\right)} b_{k} d s(2 k)+z(2 k)-z(2 k+1), \text { and } \\
r_{s, k} & =2 b_{k} d s(2 k)+z(2 k)+z(2 k+1)
\end{aligned}
$$

respectively. Note that $r_{d, k}$ and $r_{s, k}$ have the same power in the absence of $z(t)$. We then compute the correlation between them as

$$
\rho_{k} \triangleq r_{d, k} r_{s, k}^{*}=4\left|b_{k} d s(2 k)\right|^{2} e^{j\left(\beta_{k}+Q_{k} u\right)}+I_{c}(2 k),
$$

where $I_{c}(2 k)$ denotes the sum of cross correlations between the LOS path and $z(t)$ and the autocorrelation between $z(t)$, $(\cdot)^{*}$ denotes the conjugate operation.
We can then get the estimate for $u$ from $\rho_{k}$ as

$$
\hat{u}_{k}=\left(\angle\left(e^{-j \beta_{k}} \rho_{k}\right)+2 q \pi\right) / Q_{k},
$$

where $\angle\left(e^{-j \beta_{k}} \rho_{k}\right) \in[-\pi, \pi]$, and $q$ is an integer between 0 and $Q_{k}-1$. Note that in (5), there is a phase ambiguity problem as $Q_{k}$ is not directly known without prior knowledge on the AoA range. Referring to $q=0$, the directly resolvable range of equivalent AoA is $|u| \leq \pi / Q_{k}$, and the AoA is

$$
|\theta| \leq \operatorname{asin}\left(1 / Q_{k}\right) \triangleq \zeta_{\theta}\left(Q_{k}\right),
$$

where $\operatorname{asin}(x)$ is the inverse function of $\sin (x)$. That is $\zeta_{\theta}\left(Q_{k}\right)=1.5708,0.5236,0.2527$ and 0.1253 (unit: radius) for $Q_{k}=1,2,4$ and 8 respectively. The corresponding bounds for the equivalent AoA are $\zeta_{u}\left(Q_{k}\right)=\pi, \pi / 2, \pi / 4$ and $\pi / 8$. These are the constraints on the range of (equivalent) AoA that can be directly estimated for different $Q_{k}$. Beyond this range, the phase ambiguity problem arises and the value of $q$ needs to be determined using prior knowledge.

\section{B. Estimation Performance and CRLB}

Without phase ambiguity, the estimate from (5) is always more accurate for a larger $Q_{k}$, when there is only one multipath. This can be quantitatively analyzed by the CRLB, which provides a lower bound for the variance of estimation error.

From (4), the mean signal-to-interference-and-noise (SINR) can be approximated as

$$
\gamma_{k}=\frac{16\left|b_{k} d s(2 k)\right|^{4}}{\sigma_{c}^{2}},
$$

where $\sigma_{c}^{2}$ is the variance of $I_{c}(2 k)$.

Let $\gamma_{0}=|d s(2 k)|^{2} / \sigma_{z}^{2}$ be the base-SNR, the power ratio between the signal arriving at an antenna element and the noise. In the absence of NLOS paths, we can get

$$
\gamma_{k}=\left(\frac{1}{\left|b_{k}\right|^{2} \gamma_{0}}+\frac{1}{4\left|b_{k}\right|^{4} \gamma_{0}^{2}}\right)^{-1} .
$$

Initially, for a uniform $\mathrm{BF}$ response over all directions $[-\pi, \pi]$, $\left|b_{k}\right|$ is approximately $1 / \sqrt{2}$, and we get

$$
\gamma_{k}=\frac{2}{\gamma_{0}}+\frac{1}{\gamma_{0}^{2}} \text {. }
$$

Assume that $I_{c}(2 k)$ in (4) follows Gaussian distribution. We can now formulate (4) as a problem of estimating $e^{j Q_{k} u}$ in the presence of AWGN with mean 0 and variance $\sigma_{c}^{2}$. Note that we do not need to know the value of $4\left|b_{k} d s(2 k)\right|^{2}$ to estimate $u$. Hence the likelihood function $p\left(\rho_{k} \mid u\right)$ can be represented as

$$
\begin{array}{r}
p\left(\rho_{k} \mid u\right)=\frac{1}{2 \pi \sigma_{c}} \exp \left(\frac{1}{2 \sigma_{c}^{2}}\left(\rho_{k}-4\left|b_{k} d s(2 k)\right|^{2} e^{j Q_{k} u}\right) .\right. \\
\left.\left(\rho_{k}-4\left|b_{k} d s(2 k)\right|^{2} e^{j Q_{k} u}\right)^{*}\right) .
\end{array}
$$

The CRLB of the estimation error $e_{u} \triangleq \hat{u}-u$ is then given by [5]

$$
\begin{aligned}
\operatorname{CRLB}\left(e_{u}\right) & =\left(\mathrm{E}\left[-\frac{\partial^{2} \ln p\left(\rho_{k} \mid u\right)}{\partial u^{2}}\right]\right)^{-1} \\
& =\left(2 Q_{k}^{2} \gamma_{k}\right)^{-1} .
\end{aligned}
$$


It is clear that the CRLB for a doubled $Q_{k}$ value is equivalent to a $6 \mathrm{~dB}$ SINR gain, given that the $\mathrm{BF}$ gain and interference power are the same for them. We will further see in Section III-C that the $\mathrm{BF}$ gain for a larger $Q_{k}$ is typically also larger. Therefore, we shall use a larger $Q_{k}$ whenever the phase ambiguity problem can be resolved.

\section{Design BF Vector $\mathbf{w}_{1}(t)$}

We can pre-generate the $\mathrm{BF}$ vectors for different $Q_{k}$ values using, e.g., the iterative least-squares (ILS) method [6]. Since we generally only specify the magnitude $\mathbf{P}_{k}$ of the desired BF response, there are $N / 2$-degrees of freedom for determining its phase vector. The ILS method iteratively finds a near-optimal solution for the phase vector of the desired BF response, while computing the LS solution for $\mathbf{w}_{1}(2 k)$.

With given AoA ranges, we can pre-generate these BF vectors to enable fast on-line operation. For example, if we know the range of AoA $\left[-\delta_{\theta}, \delta_{\theta}\right]$ and hence the equivalent AoA $\left[-\delta_{u}, \delta_{u}\right]$, we can pre-generate $\mathrm{BF}$ pointing at zero angle with the desired BF waveform. Exemplified BF waveforms with different $\mathbf{P}_{k}$ for different $Q_{k}$, generated by the ILS algorithm, are shown in Fig. 1a. Fig. 1b presents the waveforms when the same $\delta_{u}$ is specified for different $Q_{k}$ values. From both figures, We can see that these waveforms have a reasonable uniform coverage over the range of interest, and a larger $Q_{k}$ typically leads to a higher BF gain in the area of coverage. Therefore, whenever the phase ambiguity can be resolved, a larger $Q_{k}$ is preferred.

Due to the preference of using a larger $Q_{k}$, we only need to pre-generate a limited number of $\mathrm{BF}$ vectors with $\mathrm{BF}$ waveform covering a certain AoA range for each $Q_{k}$. We can then choose the vector with the coverage range larger than and closest to the desired one. When the pointing direction needs to be changed, we can multiply a linear phase shifting sequence to the selected $\mathrm{BF}$ vector, to shift the BF pointing direction from zero degree to the centre of the desired one [7]. The shifted BF vector is then used as $\mathbf{w}_{1}(t)$.

\section{Acquisition and Tracking Algorithms}

We describe how to select the value of $Q_{k}$ and then apply the pre-designed BF vector for acquisition and tracking here. For acquisition, we assume there is no prior knowledge on the range of AoA; and for tracking, we assume the range of AoA is known.

1) Progressive Method for Acquisition: The basic idea of the progressive method is to get one estimate from every two measurements for one $Q$ value, and repeat this process with doubled $Q$ values progressively. Hence the AoA range to be estimated is narrowed down by half after each estimate until $Q$ reaches the maximum value. The basic progressive method includes several iterations and is described below:

S1: Start with $k=1: Q_{1}=1$ and $\beta_{1}=0$ when without any prior knowledge on $u$, that is $u \in[-\pi, \pi]$;

S2: At time $2 k$ (or iteration $k$ ): obtain the estimate $\hat{u}_{k}$ using the virtual subarray method as described in Section III-A, by letting $q=0$ in (5);
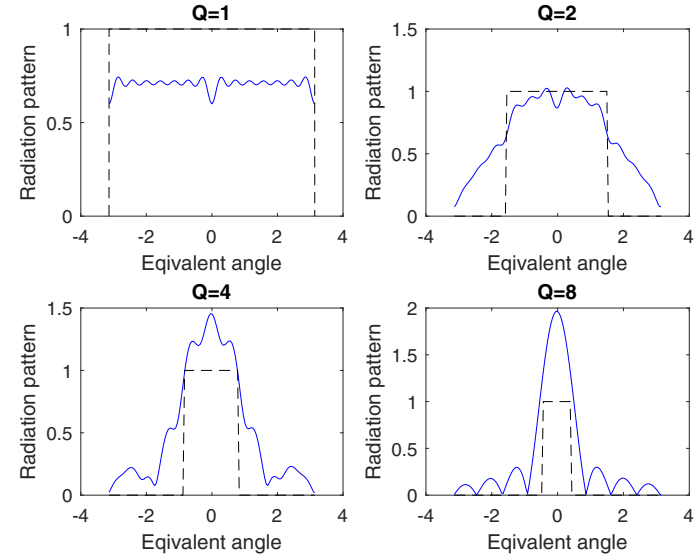

(a) Different AoA ranges (denoted by black dashed curves) are specified for different $Q_{k}$ values: $\delta_{\theta}=1.5,0.5,0.25$ and 0.125 , and $\delta_{u}=0.9975 \pi, 0.4794 \pi, 0.2474 \pi$ and $0.1247 \pi$, for $Q_{k}=1,2,4$, and 8 respectively.
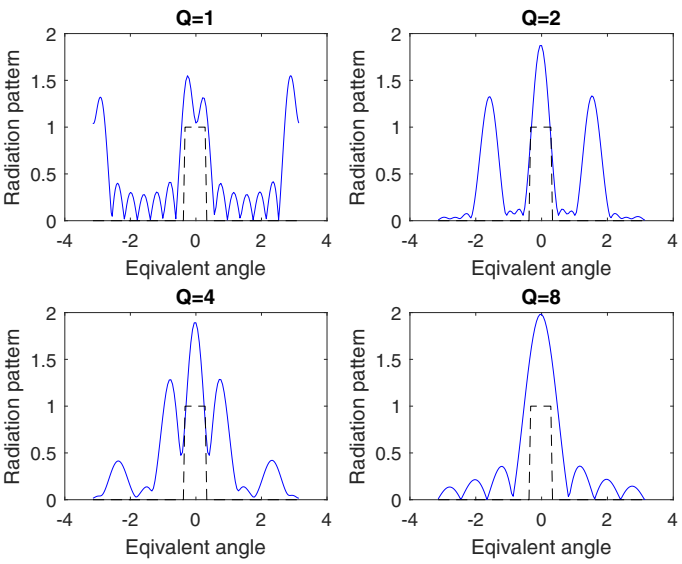

(b) The same AoA range with $\delta_{u}=0.2809$ (denoted by black dashed curves) is specified for different $Q_{k}$ values.

Fig. 1: Generated BF waveform by the first virtual subarray using the ILS algorithm.

S3: At time $2(k+1)$ (or iteration $k+1$ ): Let $Q_{k+1}=2 Q_{k}$ and $\beta_{k+1}=-Q_{k+1} \hat{u}_{k}$. Let $\mathbf{w}_{1}(2(k+1))$ be the pregenerated $\mathrm{BF}$ vector with $\delta_{u}=\pi / Q_{k+1}$;

S4: Shift the central pointing direction of the BF waveform to $-Q_{k+1} \hat{u}$ by multiplying $\mathbf{w}_{1}(2(k+1))$ with a phase shifting sequence given by $\left\{e^{-j n Q_{k+1} \hat{u}_{k}}\right\}$, where $n$ is the index of the antenna in the array, belonging to virtual subarray 1 ;

S5: Let $k=k+1$ and repeat from $\mathrm{S} 2$; until the maximum $Q$ value is reached. If the desired number of iterations are larger than the maximum $Q$ value, the maximum $Q$ is kept unchanged in the following estimation and $u$ is estimated based on the signals $e^{-j \beta_{k}} \rho_{k}$ averaged over time with a forgetting factor, e.g., 0.7.

This method implicitly assumes that each estimate of $u$ needs to identify a correct zone of width $\pi / Q_{k}$, containing the actual value of $u$, and the estimation error shall not be larger than $\pi / Q_{k}$. With this assumption and the setting of $\beta_{k+1}=-Q_{k+1} \hat{u}_{k}$ in $\mathrm{S} 3$, we limit the value of $q$ to be zero 
in the estimate of (5). So the phase ambiguity is implicitly resolved.

This method works very well when the SNR is sufficiently large so that the estimation error at time $2 k$ is rarely larger than $\pi / Q_{k}$. However, when SNR is low and the error is larger than $\pi / Q_{k}$ (named as "zoning error"), the BF waveform generated in the next iteration with a doubled $Q_{k}$ will not cover the actual AoA. The BF gain $b_{k}$ will be very small in this case, and all the rest estimates will be wrong. So we need a mechanism to rectify this problem.

One mechanism we propose here is to exploit the additional information contained in the measurements and the sequentially obtained estimates. More specifically, in S3, we change to the following processing which decides among using a doubled $Q$ value, keeping the current value, or reducing it by half in the next iteration.

C1: At time $2(k+1)$, If $\left|\rho_{k+1}\right|<\lambda_{\rho}\left|\rho_{k}\right|$ and $\left|\hat{u}_{k+1}-\hat{u}_{k}\right|>$ $\lambda_{u} \pi / Q_{k+1}$, where $0<\lambda_{\rho}<1$ and $0<\lambda_{u}<1$ are two pre-set thresholds, we conjecture that the actual AoA is out of the coverage of current BF waveform. Therefore, we will use a broader BF waveform at time $2(k+2)$. That is, we let $Q_{k+2}=Q k+1 / 2$ and $\hat{u}_{k+1}=\hat{u}_{k}$, until $Q=1$;

C2: Else, if $\left|r_{d, k+1} / r_{s, k+1}\right|+\left|r_{s, k+1} / r_{d, k+1}\right|>\lambda_{r}$, where $\lambda_{r}>2$ is a preset threshold, we conjecture that the noise in the current measurements is too large and the estimate may have a large error and should be discarded. So at time $2(k+2)$, the value of $Q$ will not be changed, and the previous AoA estimate will be used instead of the current one.

This mechanism is heuristic and not optimized, but it is found to work well at low SNRs. The thresholds are set as $\lambda_{\rho}=0.8$, $\lambda_{u}=2 / 3$, and $\lambda_{r}>3$ in the simulation.

2) Tracking: Once a relatively accurate estimate of AoA is obtained, we can enter the AoA tracking stage if the AoA changes slowly. During the tracking stage, the dynamic range of AoA needs to be known and can be determined based on, for example, the maximal relative moving speed between the transceiver and their distance.

We prefer to using a larger $Q_{k}$ as it typically leads to better estimation accuracy. Let the dynamic range of the equivalent AOA be $\delta_{u}$. The selection condition can be represented as follows:

$$
Q_{k}= \begin{cases}1, & \text { when } \delta_{u} \geq \zeta_{u}(2)=\pi / 2, \\ 2, & \text { when } \pi / 4=\zeta_{u}(4) \leq \delta_{u}<\pi / 2, \\ 4, & \text { when } \pi / 8=\zeta_{u}(8) \leq \delta_{u}<\pi / 4 \\ 8, & \text { when } \delta_{u}<\pi / 8\end{cases}
$$

If more signals are available for AoA tracking, an iterative process similar to the acquisition method can be applied, until the maximum $Q$ value is reached.

\section{Simulation Results}

We simulate a ULA with $N=16$ antenna elements. The conventional scanning method and the compressive sensing based random sweeping [4] are used as benchmarks for comparison. In the scanning method, for a given total measurement

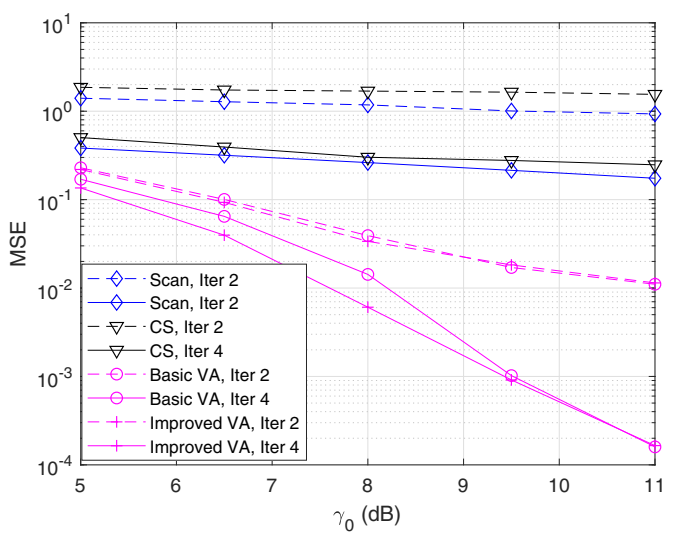

Fig. 2: MSE of estimates versus SNR.

times $K$, the ULA forms a single beam to scan $2 K$ directions uniformly distributed over the equivalent AoA range, and then the direction with the maximum received power is identified as the estimate for $u$. The CS-based method uses a dictionary of size 192 , and the grid interval is 0.0295 . The orthogonal matching pursuit (OMP) algorithm is used for CS recovery. We use the mean square error (MSE) $\sum_{m=1}^{M}\left|u_{m}-\hat{u}_{m}\right|^{2} / M$ and the mean BF gain of $\left|\mathbf{w}^{T} \alpha(\operatorname{asin}(\hat{u} / \pi))\right|$ as the performance metrics.

\section{A. Acquisition Performance}

For acquisition, the AoAs are randomly generated from a uniform distribution over the equivalent AoA $[-0.9 \pi, 0.9 \pi]$. The used BF waveforms are as shown in Fig. 1a. In the figures, the scanning method, CS method, and the basic and improved proposed acquisition schemes are denoted as "Scan", "CS", "Basic VA" and "Improved VA", respectively.

In Fig. 2, we show how the MSE varies with SNR for the estimates at Iteration 2 and 4. From the figure, we can see that the proposed VA schemes significantly outperform "Scan" and "CS". As expected, "Improved VA" improves the accuracy at a smaller SNR, and the improvement becomes negligible when SNR is sufficiently large and the "zoning error" rarely happens.

Fig. 3 demonstrates how the MSE is reduced with number of iterations increasing. When SNR is small, there is an error floor which is mainly caused by the "zoning error" at initial iterations. This error floor is removed in "Improved VA" when the SNR is large. Fig. 4 presents the cumulative density function $(\mathrm{CDF})$ of the estimation error. We can see that most estimates in "Improved VA" have very small error, particularly for iterations 3 and 4 . The MSE is enlarged by a limited number of large errors.

Fig .5 presents the gain achieved by the estimate. We can see that even at $\mathrm{SNR}=6.5 \mathrm{~dB}$ at Iteration 3 , the mean $\mathrm{BF}$ gain already approaches the maximal achievable value 16 . This is consistent with the observation in Fig. 4 which shows that most estimates have small errors. 


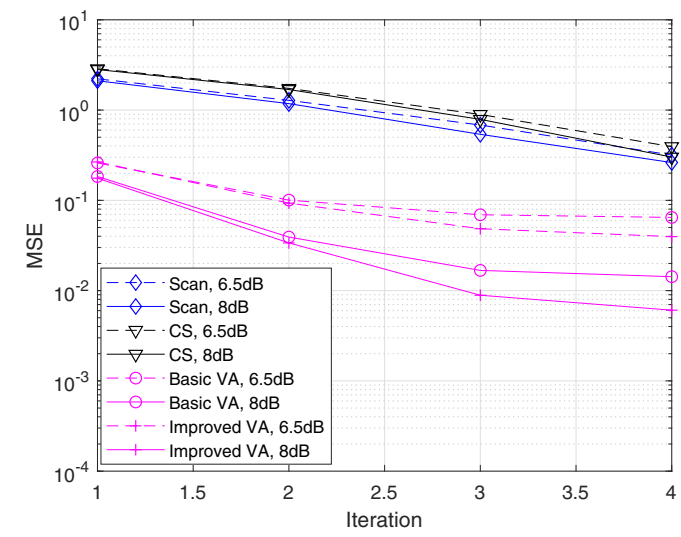

Fig. 3: MSE of estimates versus number of iterations.

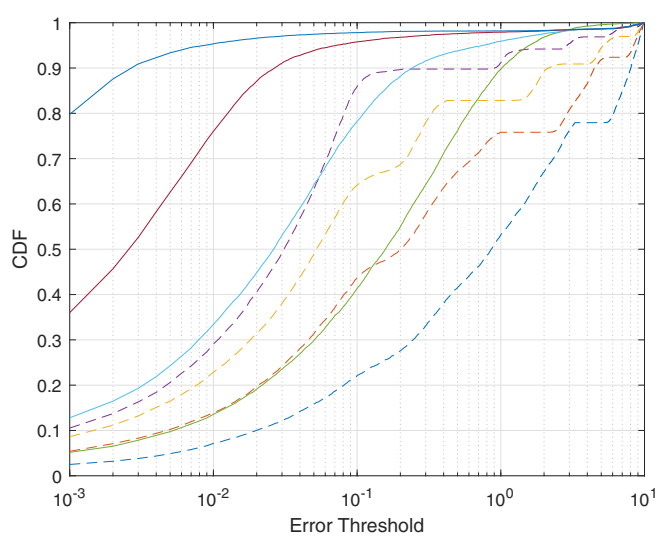

Fig. 4: CDF versus number of iterations. Solid and dashed curves are for "Improved VA" and "Scan", respectively. For each type of curves, iterations are $4,3,2,1$ from left to right.

\section{B. Tracking Performance}

In Fig. 6, we present the tracking MSE when $\delta_{u}=0.28$ is known. The equivalent AoA is randomly and uniformly generated between $\left[-\delta_{u}, \delta_{u}\right]$. The used $\mathrm{BF}$ waveforms for different $Q_{k}$ values are shown in Fig. 1b. The estimate is obtained from two measurements only without iteration. The results for scanning at two directions within this range are also plotted, together with the CRLBs. It can be seen that the proposed methods can achieve very high accuracy when the AoA range is known, and the numerical results match the CRLBs very well. The performance gap between two $Q$ values of ratio 2 is slightly larger than $6 \mathrm{~dB}$, resulted from both (10) and the BF gain.

\section{CONCLUSiOnS}

We have presented a virtual-subarray based AoA estimation scheme that can obtain AoA estimation directly using only two measurements over time. Different constructions of virtual subarray are proposed and it is shown that a construction with a larger $Q$ can lead to more accurate estimate when the phase ambiguity problem is resolved. Applications of the scheme in AoA acquisition and tracking are proposed and the proposed

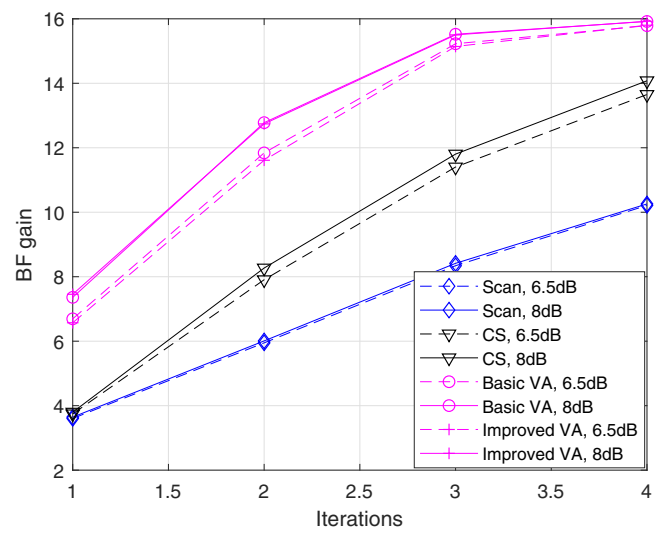

Fig. 5: BF gain versus number of iterations.

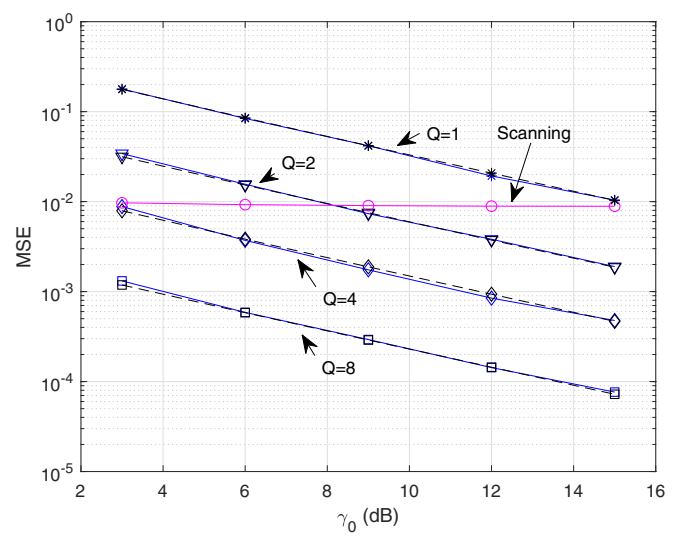

Fig. 6: MSE of estimates (solid curves) and CRLB (dashed curves) versus base-SNR for various $Q_{k}$ values.

scheme is shown to significantly outperform conventional ones in both cases. This scheme can also be extended and applied to other array systems, e.g., uniform square array and hybrid array systems.

\section{REFERENCES}

[1] J. A. Zhang, W. Ni, P. Cheng, and Y. Lu, "Angle-of-arrival estimation using different phase shifts across subarrays in localized hybrid arrays," IEEE Commun. Lett., vol. 20, no. 11, pp. 2205-2208, Nov. 2016.

[2] J. Li, Y. Sun, L. Xiao, S. Zhou, and C. E. Koksal, "Fast analog beam tracking in phased antenna arrays: Theory and performance," 2017. [Online]. Available: http://arxiv.org/abs/1710.07873

[3] S. Hur, T. Kim, D. J. Love, J. V. Krogmeier, T. A. Thomas, and A. Ghosh, "Millimeter wave beamforming for wireless backhaul and access in small cell networks," IEEE Trans. Commun., vol. 61, no. 10, pp. 4391-4403, Oct. 2013.

[4] A. Alkhateeb, G. Leus, and R. W. Heath, "Compressed sensing based multi-user millimeter wave systems: How many measurements are needed?" in Proc. IEEE Int. Conf. Acoust. Speech Sig. Process., Apr. 2015, pp. 2909-2913.

[5] X. Huang and Y. J. Guo, "MSE lower bounds for phase estimation based on overlapped gaussian distribution," in 2010 10th International Symposium on Communications and Information Technologies, Oct 2010, pp. 655-660.

[6] Z. Shi and Z. Feng, "A new array pattern synthesis algorithm using the two-step least-squares method," IEEE Signal Process. Lett., vol. 12, no. 3, pp. 250-253, Mar. 2005.

[7] J. A. Zhang, X. Huang, Y. J. Guo, J. Yuan, and R. W. Heath, "Multibeam for joint communication and radar sensing using steerable analog antenna arrays," IEEE Trans. Veh. Technol., vol. 68, no. 1, pp. 671-685, 2019. 